\title{
Dos caras de la misma moneda
}

\section{La teoría y la práctica de la comunicación en las organizaciones}

\section{Two sides of the same coin}

The theory and the practice of communication in organizations

\section{Dois lados da mesma moeda}

\section{A teoria e a prática da comunicação nas organizações

paul.capriotti@urv.cat - Editor invitado / Universitat Rovira i Virgili, España.

ORCID: https://orcid.org/0000-0002-9398-5886

En los últimos 50 años, la comunicación en las organizaciones (en sus diversas expresiones: comunicación corporativa, relaciones públicas, comunicación institucional, etc.) ha estado atravesada, como espacio académico, por la tensión entre el enfoque práctico (profesionalista) y la perspectiva teórica (academicista).

Este debate -en ocasiones, agrio- ha enfrentado muchas veces las posiciones de los profesionales de la comunicación (los prácticos) con los puntos de vista de los investigadores de las universidades (los teóricos). Por un lado, están los profesores e investigadores profesionalistas (los prácticos), quienes buscan desarrollar una actividad investigadora muy vinculada con la labor profesional en las organizaciones. Y, por otrolado, se encuentran los profesores y los investigadores comunicólogos (los teóricos), que pretenden orientar la investigación hacia cuestiones principalmente conceptuales de los diferentes aspectos de la comunicación organizacional. 
El dilema planteado ha suscitado una destacada polémica dentro del propio seno de las universidades. El sector profesional ha catalogado a la investigación en comunicación en las organizaciones como excesivamente conceptual y alejada de la práctica cotidiana, mientras que muchos investigadores del mundo académico han visto en los profesionales una pretensión puramente instrumentalista y poco reflexiva de la investigación.

Esta es una falsa oposición. Una teorización excesiva sin una búsqueda de vinculación con la actividad organizacional (ola práctica profesional) hace que la reflexión se vuelva una actividad puramente contemplativa y desligada de la vida cotidiana, como la prédica de un monje ermitaño en medio de la selva o en la punta de una montaña. Y un excesivo practicismo sin análisis y reflexión conceptual no es más que una mera acumulación de experiencias personales y casos concretos sin posibilidad de interpretación o aprovechamiento para otras situaciones, como las batallitas y anécdotas que un abuelo cuenta a sus nietos.

Falsa oposición, insisto, porque la investigación de la comunicación en las organizaciones en el seno de las universidades se puede construir mediante la combinación del análisis de los saberes profesionales, en particular, y de la comunicación pública, en general. Pero, en cualquier caso, esta investigación adquiere, por definición, un carácter reflexivo, cualificado y científico. Otra cosa es que, a partir de estas premisas esenciales, la investigación pueda tener una orientación más "conceptual" (incidiendo en la reflexión sobre los aspectos teóricos) o más "práctica" (incidiendo en el análisis de los saberes profesionales).

Así, esta doble perspectiva, esta dualidad, que en muchas ocasiones ha sido considerada como un "problema", también puede ser observada como una gran oportunidad. Una posibilidad de establecer un sincretismo investigador y combinar lo mejor de ambos mundos, buscando su mutua interdependecia y complementación.

Este volumen de InMediaciones de la Comunicación busca ser un reflejo de ello. Los ocho artículos y la entrevista publicados combinan, cada uno de ellos con sus propios matices, las reflexiones conceptuales conla práctica profesional o la actividad comunicativa en las organizaciones.

El artículo "Fake news y relaciones públicas en América Latina", de Andréia Silveira Athaydes, Alejandro Álvarez-Nobell y Gabriel Sadi, se focaliza en un tema de máxima actualidad: las noticias falsas. Los autores reflexionan sobre la preocupación que esto ha generado en los profesionales de la comunicación en las organizaciones latinoamericanas. Es un trabajo que combina el rigor académico con el manejo y el tratamiento de un gran volumen de datos generados por el Latin American Communication Monitor, lo que permite analizar los principales aspectos vinculados al fenómeno de las fake news y la consolidación de las redes sociales. 
Por su parte, Viena Medina González, Ana Almansa Martínez y Antonio Castillo Esparcia presentan el artículo titulado: "Gestión del conocimiento en las universidades. Comunicar en entornos digitales”. Allí buscan establecer puentes entre la comunicación, la importancia de las tecnologías y la gestión del conocimiento en las universidades. En este artículo hay una revisión de los conceptos clave sobrela comunicación del conocimiento, parallegar a plantear los ejes fundamentales de la relación que se establece entre ellos y aplicarlos a la gestión del conocimiento científico en las instituciones universitarias. Es un aporte interesante que se vale de la revisión y la articulación de los aspectos conceptuales, su aplicación a el ámbito universitario específico y el papel de la gestión de las tecnologías como herramientas para lograr un efectivo flujo de información del conocimiento científico.

En el artículo "Evaluación de la actitud interactiva y el nivel de diálogo de las empresas de Colombia en Facebook", Ileana Zeler profundiza en algunos aspectos centrales de la comunicación en las redes sociales de las organizaciones y, principalmente, en el uso de Facebook como un instrumento para fomentar e impulsar la interacción y el diálogo con los públicos. Zeler aplica y traslada los conceptos al estudio específico de las empresas con mejor reputación en Colombia, con el propósito de analizar su actividad en Facebook y reflexionar sobre su predisposición y su capacidad de diálogo con los públicos. La autora concluye que si bien las empresas tienen una actividad destacada en esta red social, su orientación al diálogo aún es limitada. Es un artículo que contribuye a reflexionar sobre la puesta en práctica del diálogo en las organizaciones, más allá de su incuestionable importancia teórica.

En el siguiente artículo, titulado "Del sector secundario al quinario. Comunicación interna según la actividad de la empresa: objetivos, medición y prioridades", Cristina Aced-Toledano y Susana Miquel-Segarra repasan la relevancia estratégica que tanto los académicos como los profesionales le otorgan a la interacción con los empleados y realizan un estudio cuantitativo que pone de relieve -de un modo transversal- el lugar de la comunicación interna en empresas españolas de diferentes sectores dela actividad económica. El artículo profundiza en los objetivos, las características y las estrategias de las áreas de comunicación interna delas empresas, y destaca el análisis delas herramientas que son utilizadas para evaluar su gestión, así como la reflexión prospectiva sobre la evolución de la comunicación interna en los próximos años. Es un artículo que otorga una visión panorámica de la importancia y la situación actual de la comunicación interna, tanto desde una perspectiva teórica como profesional.

Luego, en el artículo "Comunicación publicitaria en medios digitales. La gestión de la responsabilidad social en las Organizaciones del Tercer Sector”, Herlinda Ortiz Rodríguez compara la difusión publicitaria de la Responsabilidad Social Organizacional (RSO) realizada por diversas organizaciones del Tercer Sector en México, Chile, España e Inglaterra. La autora lleva adelante 
un análisis de contenido en el que busca determinar la importancia que las propias organizaciones le dan a la comunicación publicitaria como difusora de acciones de RSO, y plantea que la comunicación publicitaria aún no es una actividad destacada de difusión y que las organizaciones deberán profundizar en su correcto uso y aplicación. Este artículo permite reflexionar sobre la aportación de la comunicación publicitaria como un instrumento de difusión de las actividades de estas organizaciones, y también como una herramienta que contribuye a construir y fortalecer su reputación como organizaciones éticas y comprometidas con su entorno y su comunidad.

El artículo de Francisco Leslie López del Castillo, "Stakeholders y outcomes no intencionados en la comunicación estratégica", permite reflexionar a partir del análisis de un caso concreto (la Convención Marco de las Naciones Unidas sobre el Cambio Climático celebrada en Madrid en 2019) sobre la evaluación de las acciones no intencionales en la gestión de comunicación estratégica. López del Castillo analiza la importancia otorgada a la definición y concreción de los objetivos en los diferentes sistemas y modelos de evaluación de la comunicación en las organizaciones. A partir de eso, plantea la medición de los objetivos no definidos previamente, sino que surgen espontáneamente a partir de la evolución de una situación comunicativa concreta. Es un artículo que se detiene e incide en el análisis específico del discurso mediático, para concluir que los medios de comunicación han presentado una perspectiva discordante (el greewashing) con los objetivos principales (preocupación medioambiental) de los patrocinadores del evento.

En el artículo "Gobierno abierto en la gestión de los ayuntamientos. Política informacional e intermediación a través de las redes sociales del ayuntamiento de Sant Cugat del Vallès", Zahaira González Romo y Juan Miguel Fernández Álvarez reflexionan sobre la comunicación gubernamental 2.0 a escala local, a la vez que desarrollan un estudio de la estrategia comunicativa de un ayuntamiento español que les permite reafirmar la importancia que ha adquirido la comunicación digital en las pequeñas instituciones para lograr una adecuada difusión de sus actividades. Este artículo aporta reflexión sobre algunos aspectos claves de la comunicación de las instituciones públicas locales, y hace un estudio de caso sobre el uso de la comunicación digital y las nuevas formas de interacción social que permiten observar en qué medida los aspectos conceptuales pueden vincularse con fenómenos del día a día.

En el último artículo, titulado "Comunicación corporativa para la fidelización del cliente”, Patricia Durán Bravo, Nancy Cisneros Martínez y Raúl Pancardo Peralta proponen una visión novedosa y un modelo estratégico para diagnosticar los alcances de la comunicación corporativa y fortalecer la lealtad delos clientes. Los autores retoman diversas teorizaciones y hacen del diagnóstico y la comunicación un eje vertebrador de todo el proceso de fidelización y convierten a la gestión comunicativa en el centro del sistema integrador. Así, 
el artículo plantea un interesante modelo donde se combinan las perspectivas de la comunicación organizacional, el branding y el marketing, y pone el foco de atención en el análisis y en la gestión de las relaciones entre la organización y sus clientes.

Finalmente, la entrevista a Carlos A. Scolari es el máximo exponente de dicho sincretismo teórico-práctico. Bajo el título "Desde hace un siglo la comunicación trata infructuosamente de construir algo coherente", Scolari repasa los aspectos más destacados de las últimas tendencias en el ámbito de la comunicación y los vincula con las teorías clásicas para actualizar y repensar algunos de sus conceptos fundamentales. Reflexiona sobre el comunicador como marca, las narrativas transmedia, la ecología de los medios, el storytelling, la alfabetización transmedia y los influencers, integrando conceptos profundos y ejemplos cotidianos desde múltiples perspectivas, pero dando a la vez una visión en la que todo se relaciona con todo. En tal sentido, Scolari plantea que quizás ya hemos superado la etapa de la sociedad líquida para pasar a un nuevo estado: la sociedad gaseosa.

Como quedó mencionado, este volumen de InMediaciones de la Comunicación, centrado en el tema Tendencias y desafíos de la comunicación en las organizaciones: crisis, reputación y gestión de vínculos, busca contribuir a la reflexión conceptual sobre algunos de los aspectos relevantes de la comunicación en las organizaciones. Además, pretende vincular dichas reflexiones con el análisis de situaciones concretas de la gestión comunicativa. Un balance entre las dos caras de la misma moneda: la teoría y la práctica de la comunicación en las organizaciones.

\section{(cc) BY}

Artículo publicado en acceso abierto bajo la Licencia Creative Commons - Attribution 4.0 International (CC BY 4.0).

\section{IDENTIFICACIÓN DEL EDITOR INVITADO}

Paul Capriotti. Doctor en Ciencias de la Comunicación por la Universidad Autónoma de Barcelona, España, yLicenciado en Comunicación Social (orientación en Relaciones Públicas) por la Universidad Nacional de Rosario, Argentina. Profesor de Relaciones Públicas y Comunicación Corporativa de la Universitat Rovira i Virgili(URV) en Tarragona, España. Ha publicado diversos capítulos de libros y 3 libros: Imagen de Empresa (1992, El Ateneo), Planificación Estratégica de la Imagen Corporativa (1999, IIRP-Instituto de Investigación en Relaciones Públicas) y Branding Corporativo (2009, Libros de la Empresa); todos están disponibles en: www.bidireccional.net. Es autor de diversos artículos en revistas de reconocido prestigio a nivel nacional e internacional: Public Relations Review, Journal of Communication Management, Business and Society, Corporate Communications, Museum Management and Curatorship, Communication and Society, International Journal of Communication Management. Es Profesor Invitado en diferentes universidades españolas y latinoamericanas. 